\title{
E-manuscripta.ch: Aufbau und stetige Weiterentwicklung
}

\begin{abstract}
Von drei Schweizer Hochschulbibliotheken wurde e-manuscripta.ch als Kooperationsprojekt mit Eigenmitteln aufgebaut. Die Metadaten werden aus den verschiedenen Erschliessungssystemen bezogen und in einem vordefinierten Minimalset dargestellt. Über Schnittstellen bleibt die Plattform mit den Datenbanken verbunden. Die teilnehmenden Institutionen sind frei in der Auswahl der von ihnen zu präsentierenden Dokumente und können nach der ersten Einrichtung der Verbindung jederzeit Digitalisate auf die Plattform laden. Die Plattform wird stetig ausgebaut, einerseits durch die Integration von neuen Partnerinstitutionen und andererseits durch technische Weiterentwicklungen. Eine grosse Neuerung wird die Einrichtung des Transkriptionstools darstellen. Mit der Weiterentwicklung des bestehenden Moduls um ein formalisiertes Redaktionskonzept wird das Angebot auf eine freie Teilnahme ausgerichtet unter Einhaltung gewisser Richtlinien, was auch als Grundlage für zukünftiges Crowdsourcing dient.
\end{abstract}

\section{Aufbau und Einrichtung der Plattform}

Am 13. März 2013 ist die Plattform für digitalisierte Quellen aus Schweizer Bibliotheken und Archiven unter dem Namen „e-manuscripta.ch“ online gegangen. Aufgebaut wurde sie mit Eigenmitteln von den drei Hochschulbibliotheken ETHBibliothek, Universitätsbibliothek (UB) Basel und Zentralbibliothek (ZB) Zürich. Dieselben drei Hochschulbibliotheken waren bereits bei der Einrichtung von e-rara.ch im Rahmen des Projekts e-lib.ch, der Elektronischen Bibliothek Schweiz, beteiligt. ${ }^{1}$ Sie wollten die mit der Software und der technischen Infrastruktur gesammelten Erfahrungen nutzen, um eine ähnlich aufgebaute Präsentationsmöglichkeit für verschiedenartige handschriftlich hergestellte Dokumente zu schaffen und auf diese Weise das digitale Angebot auf nationaler Ebene zu vervollständigen.

Die Organisationsstruktur ist durchaus vergleichbar mit der von e-rara.ch. Die Betreuung der technischen Infrastruktur wurde wieder von der ETH-Biblio-

1 Franziska Geisser: e-rara.ch: ein Schweizer Digitalisierungsprojekt mit internationaler Ausstrahlung. In: arbido 3/2011, S. 23-26. In derselben Ausgabe befinden sich weitere Artikel zu e-lib.ch und dessen Teilprojekten.

2 Open Access. (C) Alexa Renggli, publiziert von De Gruyter. (c) BY-NC-ND Dieses Werk ist lizenziert unter der Creative Commons Attribution-NonCommercial-NoDerivatives 4.0 Lizenz. 
thek übernommen, um zu gewährleisten, dass die Digitalisate auf ähnlichen Wegen auf die Plattform gelangen und auch die gleichen Speicheranlagen zur Verfügung stehen. Die Gesamtkoordination für das Aufbauprojekt lag bei der Projektleiterin Eva Hanke in der ZB Zürich. Bei ihr lief die Organisation der verschiedenen Teilprojekte zusammen, in welchen der Betrieb vorbereitet und eingerichtet wurde. Nach der Aufschaltung wurde die Projektleitung in die zentrale Geschäftsleitung für den administrativen Teil sowie für die Koordination der gesamten Abläufe umgewandelt.

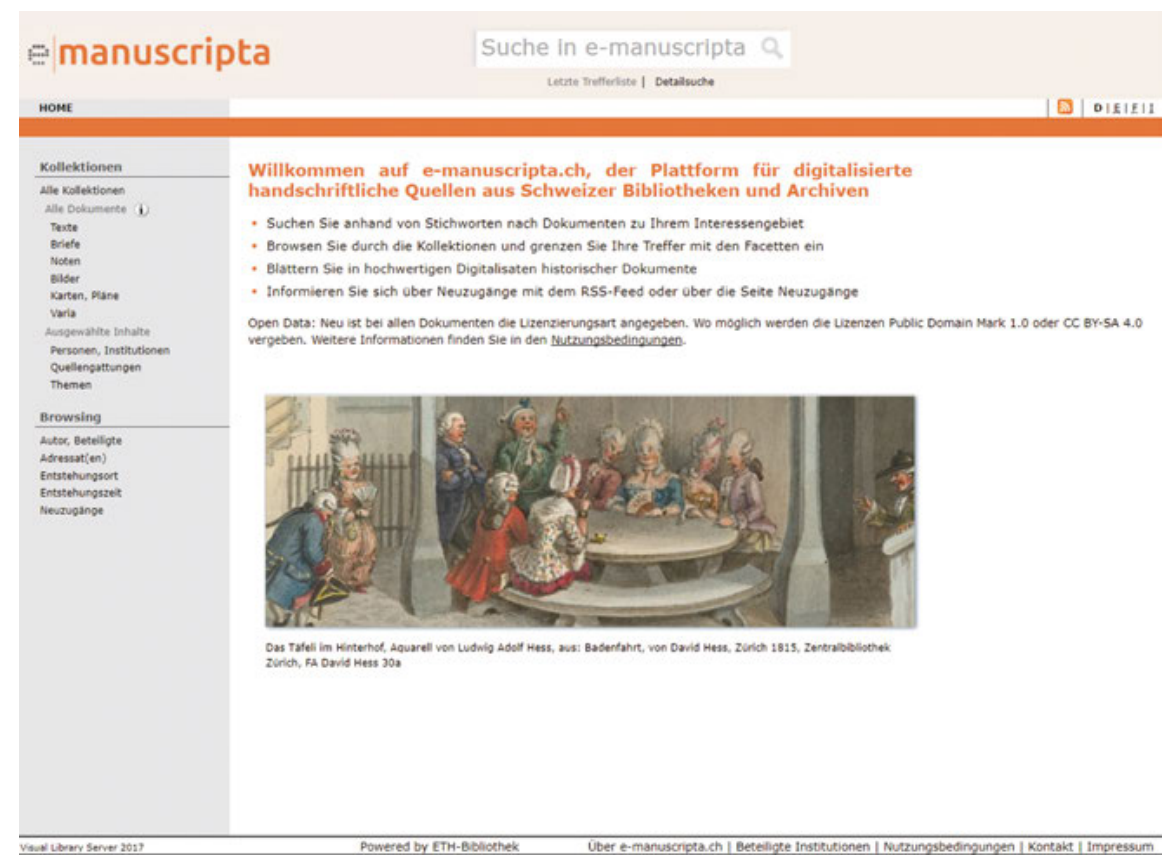

Abb. 1: Einstiegsseite e-manuscripta.ch

Das Erscheinungsbild der neuen Plattform wurde ebenfalls in enger Anlehnung an e-rara.ch gestaltet. Die Navigation erfolgt im bereits bewährten Stil über Browse-Indizes auf der linken Seite und Facetten zur Eingrenzung der Suche am rechten Rand. Für den sichtbaren Unterschied sorgt ein eigenes Logo zusammen mit abgestimmter Farbgebung. Ein wichtiges Element für den individuellen Auftritt der einzelnen Institutionen stellt eine eigene Präsentationsseite dar, auf welcher die Kontaktdaten samt eines Links auf die präsentierten Bestände zu finden sind. Als Erkennungsmerkmal für den separaten Aufritt werden den grösseren Institutionen eigene Balkenfarben zugeteilt. Sämtliche eingerichteten Sei- 
ten der Plattform liegen nicht nur in deutscher Sprache, sondern auch in Französisch, Italienisch und Englisch vor. Dadurch erhalten die präsentierten Titel nun auch weltweit grosse Beachtung und sind leicht zugänglich.

Während die Facetten und Browse-Indizes von e-rara.ch sehr ähnlich übernommen wurden, gibt es im Bereich der Kollektionen grössere Abweichungen und eine stärkere Berücksichtigung der unterschiedlichen Dokumentenarten. Statt der Hervorhebung der Erscheinungszeit wie bei den Schweizer Drucken auf e-rara.ch wurden die Dokumenttypen „Texte“, „Briefe“, „Noten“, „Bilder“, „Karten/Pläne“ und „Varia“ als primäre Unterscheidungsmerkmale ausgewählt. Das soll eine erste Orientierung bieten, welche Arten von Dokumenten zu finden sind.

Eine besondere Herausforderung stellte die Einbindung der verschiedenen Datenbanken dar, in welchen die handschriftlichen Dokumente erschlossen werden. In sorgfältiger Vorbereitungsarbeit wurde ein minimales Set von Metadaten erstellt, das auf der Plattform angezeigt werden soll. Mit einer Beschränkung auf die wesentlichen Angaben zu den Titeln wurde sichergestellt, dass es sich um Felder handelte, die in allen Verzeichnissen gefunden und sinnvoll genutzt werden können. Auf diese Weise wurden die beiden auf MARC21 basierenden Aleph-Datenbanken der UB Basel und der ZB Zürich angebunden, aber auch die mit dem Programm CMI STAR nach ISAD(G)-Normen eingerichtete Archivdatenbank des zur ETH-Bibliothek gehörenden Hochschularchivs der ETH. Auf die Anzeige von ganzen Tektonikbäumen wurde verzichtet, aber es werden gewisse übergeordnete Strukturen sichtbar und die Digitalisate eines ganzen Bestandes können aufgerufen werden.

Nach Festlegung der Felder in einem Metadatenmapping wurden die Datenbanken über Schnittstellen wie Z39.50 oder OAI-PMH mit der Plattform verbunden, damit die Metadaten jederzeit wieder auf den aktuellen Stand gebracht werden können. Dank dieser direkten Verbindung ist es möglich, in relativ schneller Abfolge eine grosse Zahl von Titeln auf die Plattform zu laden.

Während die Titel für die Präsentation auf einer zentralen Plattform zusammengeführt werden, finden die Produktion der Digitalisate und die Vorbereitungsarbeiten für die Aufschaltung dezentral in den einzelnen Institutionen statt. Um eine möglichst einheitliche Bildqualität zu erhalten, gab es zwischen den beteiligten Digitalisierungszentren einen intensiven Austausch über die Festlegung von allgemein gültigen Standards, die in einer Best Practice festgehalten wurden. Diese Richtlinien orientieren sich an den DFG-Praxisregeln. ${ }^{2}$ Die Doku-

2 http://www.dfg.de/formulare/12_151/12_151_de.pdf. Dieser Link wurde am 26.03.2018 überprüft. 
mente werden grundsätzlich einseitig mit mindestens 300dpi und einem Farbkeil gescannt. Als Dateiformat bildet TIFF den Ausgangspunkt, von welchem an der ETH-Bibliothek die JPEG- und PDF-Derivate erstellt werden. Es waren auch Absprachen $\mathrm{zu}$ treffen über die bei handschriftlichen Dokumenten viel stärker variierenden Seitengrössen oder die Ausrichtung der Schrift.

Die Vorbereitungsarbeiten vor der Publikation sind in der jeweiligen Institution im Visual Library Manager durchzuführen. In diesem Programm werden die Digitalisate erstmals zusammen mit den Metadaten sichtbar und können nochmals auf Vollständigkeit und Bildqualität kontrolliert werden. Vor der Freigabe besteht zudem die Möglichkeit, grössere Titel in Abschnitte oder Kapitel zu strukturieren. Dies soll einerseits der besseren Übersicht dienen, bietet aber andererseits auch den Vorteil, dass die einzelnen Teile separat in kleineren PDFs heruntergeladen werden können. Für diese Arbeiten war bereits für e-rara.ch eine Anleitung erstellt worden, die nun, um den speziellen Eigenheiten von Briefen und Noten gerecht $\mathrm{zu}$ werden, sachdienlich erweitert werden musste. Weitere Probleme ergeben sich durch Handschriften, die oftmals keine deutlichen Kapitelüberschriften und unregelmässige Seitennummerierungen aufweisen können. In solchen Fällen bietet die Wegleitung jeweils eine willkommene Orientierung und leistet Unterstützung beim Bestreben, den hohen Standard auch für verschiedenste Spezialfälle adäquat beizubehalten.

Nach Abschluss der Strukturierungsarbeiten erfolgt jeweils die Freischaltung, wodurch die Titel umgehend online auf der Plattform sichtbar werden. Am besten zu verfolgen ist der Zuwachs in der Browsingkategorie „Neuzugänge“, die auch als RSS-Feed abonniert werden kann. Bevor die Plattform für die Öffentlichkeit benutzbar wurde, bereiteten die Strukturierenden in den drei Partnerinstitutionen die ersten 10’000 Titel im Hintergrund vor und schalteten sie frei. Mit der Eröffnung am 13. März 2013 wurde e-manuscripta.ch gleichsam zum Schaufenster für den reichhaltigen handschriftlichen Quellenbestand, der in Schweizer Bibliotheken und Archiven aufbewahrt wird.

\section{Konsolidierung des Betriebs}

Für den Betrieb wurde als zentrales Element festgelegt, dass die Plattform von den Partnerinstitutionen kooperativ betreut wird. Die Koordination findet hierzu in der Geschäftsstelle in der ZB Zürich statt. Alle aktiven Partner haben die gleiche Stimmkraft, entscheiden gemeinsam über den Ausbau und die Weiterentwicklungen und teilen die Betriebskosten gleichmässsig untereinander auf. Passive Partner kommen nur für die von ihnen generierten Volumenkosten auf und müssen mit einem Partner assoziiert sein und von diesem betreut werden. 
Waren die Schnittstellen erst einmal eingerichtet, konnte die Befüllung der Plattform gleich nach der Eröffnung richtig Fahrt aufnehmen. In allen drei Partnerinstitutionen lagen schon grössere Vorräte an digitalisierten Dokumenten bereit, um aufgeschaltet zu werden. Nach zwei Jahren Betriebszeit waren Ende 2014 20’000 Titel online zugänglich und zeigten das breite Spektrum der Bestände mit Briefen, Textsammlungen, Noten und Archivmaterialien aus verschiedenen Jahrhunderten. Die Auswahl der Inhalte bleibt ganz den einzelnen Institutionen überlassen. Endlich konnten Dokumente von Erasmus von Rotterdam, Richard Wagner und Arnold Escher von der Linth, die schon seit Längerem gescannt waren, online publiziert werden. Dank dem grossen Lotteriefondsprojekt DigiTUR war es insbesondere in der ZB Zürich möglich, die schon lange gewünschte Digitalisierung der Korrespondenz von Johann Jakob Scheuchzer durchzuführen und gleich auf e-manuscripta.ch zu präsentieren. Für DigiTUR war es wiederum ein grosser Vorteil, dass die Plattform zum Projektstart 2013 fertig eingerichtet und auch der hausinterne Workflow bereits erprobt war. Entsprechend führte die erhöhte Produktionskapazität zu einem rasanten Anstieg an aufgeschalteten Titeln.

Um die kontinuierlich wachsende Menge an Dokumenten angemessen zu präsentieren, entschieden sich die Partnerinstitutionen, neben der nach Dokumenttyp aufgebauten Unterscheidung auch thematische Kollektionen einzurichten, in welchen die Titel einem bestimmten Quellentyp oder dem Umfeld einer Person oder Familie zugeordnet werden können. In einem Ausschuss mit Vertretungen aus allen drei Partnerinstitutionen wurden die ersten Kollektionen definiert und zur Einrichtung vorbereitet, inklusive kurzen erläuternden Texten in vier Sprachen. Jeder Titel kann bei Bedarf auch nachträglich noch einer dieser Kollektionen zugeteilt werden. Nach einer ersten Gruppe von Kollektionen, die im Februar 2014 in Betrieb genommen worden sind, sollen weitere thematisch zusammengehörende Inhalte auf diese Art hervorgehoben werden. Bei Bedarf wird der zuständige Ausschuss jeweils die neuen Vorschläge begutachten und zur Einrichtung freigeben.

Ein wichtiger Schritt zu einer noch grösseren Vielfalt auf der Plattform stellte die Entscheidung der Graphischen Sammlung der ZB Zürich dar, ihre Bilder auf den beiden Plattformen e-rara.ch und e-manuscripta.ch zu präsentieren. Ab 2015 wurde ein Workflow entwickelt, um die internen Abläufe im Haus für die zahlreichen Titel zu regeln. Kurz danach kamen die ersten Bilder in der entsprechenden Kollektion zum Vorschein. Zum gegenwärtigen Zeitpunkt beträgt der Anteil der Bilder an der gesamten Anzahl aufgeschalteter Titel 35\%, wobei es sich jedoch praktisch immer um einen einzigen Scan handelt. Gerade bei diesem Dokumenttyp erweist sich zudem die hohe Qualität der aufgeschalteten Digitalisate als grosser Vorteil, kommen doch die Ansichten und Ölporträts von Künstlern wie 
Johann Heinrich Füssli, Johann Rudolf Schellenberg oder David Hess dadurch besonders schön zur Geltung.

Ging es in der Anfangsphase des Betriebs noch primär darum, die Plattform mit Beständen aus den drei am Aufbau beteiligten Institutionen zu füllen, erschienen gegen Ende des Jahres 2014 zum ersten Mal die Bestände von neuen Mitgliedern: Die Zentral- und Hochschulbibliothek Luzern und das Schweizerische Wirtschaftsarchiv traten der Plattform als passive Partner bei. Sie werden von der UB Basel als aktivem Partner betreut. Da beide bereits Mitglieder des HAN-Katalogverbunds waren und dementsprechend ihre Bestände in der gleichen Datenbank erfasst haben wie die UB Basel, konnte auf das bestehende Metadatenmapping zurückgegriffen werden. Die Digitalisate werden von der UB Basel über die gleiche Verbindung an die ETH-Bibliothek geschickt, die für ihre eigenen Uploads eingerichtet worden war. Es ist zu erwarten, dass nach und nach weitere Mitglieder aus dem HAN-Verbund an einer Teilnahme interessiert sein werden.

\section{Technische Weiterentwicklungen}

\section{Nutzungsbedingungen}

In einem gemeinsamen Grundsatzentscheid beschlossen die beiden Steuerungsgremien von e-rara.ch und e-manuscripta.ch im April 2015, die aktuell bestehenden Nutzungsbedingungen für beide Plattformen abzulösen durch eine Public Domain Mark für die Digitalisate gemeinfreier Werke und eine CC-BY-SA-Lizenz für die Digitalisate urheberrechtlich geschützter Materialien. Bei jedem Titel sollte fortan direkt ein Hinweis stehen, wie die Digitalisate genutzt werden können. Auf diese Weise wird gleichzeitig verdeutlicht, dass der grösste Teil der aufgeschalteten Dokumente frei nutzbar ist als Open Data.

Die Einführung der neuen Nutzungsbedingungen für die beiden Plattformen lag bei der Gesamtkoordination von e-rara.ch. Die Arbeiten umfassten zunächst die technischen Abklärungen mit Semantics. Die Texte der einzelnen Lizenzen sollten möglichst automatisiert eingespielt werden und der Austausch der bisher vorhandenen PDF-Deckblätter war zu programmieren. Für e-manuscripta.ch wurde im Rahmen der Ausarbeitung der Lizenztexte eine dritte Variante von Nutzungsregelung hinzugenommen, die verwendet werden kann, wenn bei einem urheberrechtlich geschützten Titel zusätzlich noch Rechte bei Dritten liegen.

Sobald die Texte und technischen Einrichtungen vorbereitet waren, galt es, die bereits aufgeschalteten Titel mit den entsprechenden Lizenzen auszustatten. 
Zuerst wurden die kleineren Mengen mit eingeschränkten Rechten eingerichtet, bevor die Umstellung beim grossen Rest stattfand. Am 01. Dezember 2016 war es dann soweit, dass die neue Regelung in Kraft treten konnte und mit Meldungen auf den Homepages der beteiligten Institutionen sowie auf den beiden Plattformen selbst angekündigt wurde.

\section{Transkriptionstool}

Bald nach der Aufschaltung von e-manuscripta.ch entstand bei den teilnehmenden Institutionen grosses Interesse, einen Ausbau der Plattform um das bereits existierende Modul „Autographenerschliessung“ in Betracht zu ziehen. Eine für Nutzende nicht sichtbare Testversion wurde schon im Jahr der Inbetriebnahme installiert, was den Partnern ermöglichte, erste Erfahrungen damit zu sammeln und die vorhandenen Funktionalitäten kennenzulernen. In den weiteren Abklärungen zum Projekt als Ganzem wurde jedoch bald deutlich, dass sich die Beschaffungskosten in einer Höhe bewegen, die nur mit Hilfe von Fördermitteln $\mathrm{zu}$ bestreiten sein würde.

Ein erster Anlauf mit einem Projektantrag bei SUK P-2 „Wissenschaftliche Information“ blieb erfolglos, aber die Erfahrungen daraus halfen, die Ziele konkreter zu formulieren und das Projekt im Kontext vergleichbarer Vorhaben auf internationaler Ebene zu positionieren. Zur gleichen Zeit erhielten mehrere grössere Editionsprojekte Fördergelder vom Schweizerischen Nationalfonds, um ihre Texte in digitaler Form zu präsentieren. Während für die ganz grossen Projekte die Einrichtung einer separaten Forschungsinfrastruktur im Vordergrund steht, bleibt es für viele Transkribierende in kleineren Projekten ein wichtiges Anliegen, dass ihre Texte in einer langfristig ausgerichteten Umgebung präsentiert werden können.

Bei der Erarbeitung des zweiten Antrags, der im August 2015 unter dem Titel „e-manuscripta.ch: Nachnutzung für die Wissenschaft - Der Weg zum Volltext“ eingereicht wurde, kristallisierte sich heraus, dass e-rara.ch und e-manuscripta. ch je eigene, aber aufeinander abgestimmte Projekte zur Generierung von Volltexten einreichen. Bei e-rara.ch wurde die Einführung von OCR angestrebt und für e-manuscripta.ch das Transkriptionstool mit Erweiterung der Funktionen um ein formalisiertes Redaktionskonzept. Im Vorfeld der Ausarbeitung der beim Infrastrukturförderprogramm SUK P-2 „Wissenschaftliche Information“ von swissuniversities zu stellenden Anträge wurden die Ziele abgestimmt mit anderen sich in der Planungsphase befindenden Vorhaben. Auch der Kontakt mit den Projektverantwortlichen der Plattform für digitale Editionsprojekte wurde gepflegt, um möglichst nach Synergien und Abgrenzungen zu suchen. 
Da die konkreten Bedarfsabklärungen bei verschiedenen Editionsprojekten längere Zeit in Anspruch nahmen, wurde im August 2015 zuerst der Antrag von e-rara.ch eingereicht mit Verweis auf den später folgenden von e-manuscripta.ch. Die Nachricht von der Genehmigung des OCR-Projekts lieferte die entscheidende Motivation, den Antrag für das Transkriptionstool fristgerecht auf die nächste Förderrunde hin fertigzustellen und einzureichen. Am 04. Juli 2016 hat der Lenkungsausschuss von SUK P-2 „Wissenschaftliche Information“ den Antrag genehmigt und die Auszahlung der beantragten Fördermittel unter gewissen Auflagen zugesprochen. An dem für ein Jahr bewilligten Projekt beteiligt sind die drei Partnerinstitutionen von e-manuscripta.ch: die ETH-Bibliothek, die UB Basel und die ZB Zürich. Die Projektleitung ist an der ZB Zürich angesiedelt.

Mit dem Projekt „e-manuscripta.ch: Nachnutzung für die Wissenschaft - Der Weg zum Volltext“ wird angestrebt, dass es durch die Transkribierenden zu einer Anreicherung der Texte mittels Eingabe von Anmerkungen und Erläuterungen kommt sowie $\mathrm{zu}$ einer besseren Auffindbarkeit der Inhalte durch Anzeige der Suchbegriffe auch im Volltext. Zum Transkribieren können sämtliche auf der Plattform veröffentlichten Digitalisate frei ausgewählt werden. Innerhalb eines ganzen Bandes kann demnach auch eine einzelne Seite bearbeitet werden oder es können mehrere Transkribierende gleichzeitig an verschiedenen Stellen darin arbeiten, was als Übung in einem Seminar gut denkbar wäre.

Um bei derart grossen Freiheiten dennoch eine gewisse Qualität zu gewährleisten und grobe Missbräuche $\mathrm{zu}$ vermeiden, ist es unerlässlich, mit einem Redaktionskonzept die nötigen Rahmenbedingungen zu schaffen. Die wichtigsten Elemente dabei sind das Erstellen eines Benutzerkontos zur Identifikation samt Kenntnisnahme der Nutzungsbedingungen und Transkriptionsrichtlinien sowie eine Kontrolle der Texte vor der Freischaltung durch eine Redaktion in der jeweils besitzenden Institution. Im Gegenzug kommen diese Reglementierungen auch den teilnehmenden Transkribierenden zugute, erhalten sie doch damit die Gewähr, dass ihre Beiträge in einem vertrauenswürdigen Umfeld erscheinen und die eigenen Texte nicht jederzeit wieder von Dritten geändert werden können. Mit diesen Elementen wurden wesentliche Grundlagen für das Crowdsourcing geschaffen. So können beispielsweise bei einem gezielten Aufruf zur Bearbeitung konkreter Materialien viele Personen gleichzeitig auf der Plattform wirken und es sind trotzdem alle Abläufe kontrollierbar.

\section{Ausbau mit neuen Partnern}

Nach einer ersten Phase der technischen Konsolidierung des Betriebs und des inhaltlichen Ausbaus sowie der Einrichtung der Nutzungsbedingungen konnte an 
eine erste grössere institutionelle Erweiterung gedacht werden. Die Anfrage der Schweizerischen Nationalbibliothek im Jahr 2016, ob das Literaturarchiv seine Bestände auf e-manuscripta.ch präsentieren könnte, kam da zu einem günstigen Zeitpunkt. Zusätzlich interessant war diese Anfrage, da die Dokumente in einer gut ausgebauten Scope-Datenbank erschlossen werden.

Da bis anhin noch nie eine Scope-Datenbank in Visual Library eingebunden worden war, mussten die Möglichkeiten des Metadatenbezugs von Grund auf erörtert werden. In regem Austausch mit den technischen Spezialisten und Kennern der Metadaten in Bern fand Semantics eine Lösung, wie via OAI-PMHSchnittstelle auf die Datenbank zugegriffen werden kann, um die Metadaten abzuholen. Mit einem sorgfältigen Abgleich der Daten mittels eines Metadatenmappings konnten die relevanten Felder auf e-manuscripta.ch abgebildet werden. Am 11. April 2017 wurde der neue Client auf dem Produktivserver aktiviert und die ersten Stücke aus dem Nachlass von Rainer Maria Rilke konnten veröffentlicht werden.

Während sicher noch weitere schöne Bestände aus dem Literaturarchiv folgen werden, steht die eingerichtete Datenstruktur nun auch für andere interessierte Institutionen, die mit dem Scope-System arbeiten, zur Verfügung. Insbesondere eröffnen diese neuen Möglichkeiten vielversprechende Perspektiven für die zahlreichen Archive, deren Bestände in einem entsprechenden Verzeichnis erschlossen worden sind. Mit der Nationalbibliothek stösst nun erstmals ein neuer aktiver Partner zu den drei Institutionen, die den Aufbau und den Beginn der Betriebsphase zusammen bestritten haben, hinzu und wird die weiteren Entwicklungen der Plattform ab diesem Zeitpunkt mitgestalten.

Für kleinere Institutionen stellt weiterhin der HAN-Verbund einen besonders gut geeigneten Ausgangspunkt für die Teilnahme an e-manuscripta.ch dar. Wenn grundsätzlich zu wenige Dokumente zur Präsentation vorhanden sind, um sich an den Infrastrukturkosten als aktiver Partner zu beteiligen, bietet sich dieser Weg als besonders sinnvolle Lösung an. Die einzelnen Stücke können in einem sorgfältig aufgebauten und fachmännisch betreuten Katalog erschlossen werden und gleichzeitig bekommen die Institutionen die Möglichkeit, ihre Bestände auf e-manuscripta.ch über eine bereits existierende Datenbankverbindung auf sehr unkomplizierte Weise zu präsentieren. Dieser mittlerweile gut erprobte Weg wurde 2017 mit der Universitätsbibliothek Bern ein weiteres Mal erfolgreich umgesetzt, während bereits noch andere im HAN-Verbund vertretene Institutionen ihr Interesse an einer baldigen Teilnahme angemeldet haben. 


\section{Zukünftige Herausforderungen}

Da die allgemeine technische Entwicklung stets weiter voranschreitet und laufend neue Möglichkeiten bietet, gibt es auch für die Plattform e-manuscripta.ch zahlreiche Perspektiven für weitere Optimierungen. Im Rahmen der vorhandenen Kapazitäten wird versucht, die stattfindenden Veränderungen $\mathrm{zu}$ verfolgen und soweit sinnvoll und möglich auch für die Plattform nutzbar zu machen. Primär handelt es sich dabei um weitere technische Verbesserungen, welche den Austausch und die Referenzierbarkeit vereinfachen sollen. Ein besonderes Desiderat für die Forschung stellt eine seitengenaue, permanent gültige Verlinkung dar. Hierfür sind Abklärungen im Gange, wie ausgehend vom bereits für jeden Titel generierten persistenten DOI-Link jeder Scan mit einer eigenen Nummer identifiziert werden kann. Ähnlich drängend ist die Implementierung einer IIIF-Schnittstelle, damit auch die Titel von e-manuscripta.ch mit den dazugehörenden Bildinformationen in anderen virtuellen Sammlungen und technischen Umgebungen (Viewer) für die weitere Bearbeitung zur Verfügung stehen. ${ }^{3}$ Mittlerweile sind weltweit bereits etwa 350 Millionen Titel über eine IIIF-Schnittstelle verfügbar, was dieses Austauschformat zum neuen Standard für den Transfer von online zugänglichen Bildern oder Texten werden lässt.

Mit der Weiterentwicklung des Transkriptionstools entsteht eine vielversprechende Grundlage für die Produktion von durchsuchbaren Volltexten. In weiteren Ausbauetappen ist vorgesehen, den Import von grösseren Textmengen zu ermöglichen und auf diese Weise bereits vorhandene oder allenfalls maschinell generierte Transkriptionen auf direktem Wege und unter Beibehaltung von möglichst vielen Formatierungen den Digitalisaten an die Seite zu stellen. Derartige Schnittstellen einzurichten, setzt eine grosse Menge an Testdaten voraus und erfordert einen hohen zeitlichen Aufwand, der erst nach Abschluss des momentan laufenden SUK P-2-Projekts „Wissenschaftliche Information“ in einem allfälligen neuen Projekt in Erwägung gezogen werden kann.

Während die Plattform mit ihren aktuell 65’000 Titeln schweizweit bereits einen zentralen Einstieg in einen grossen Briefbestand und ein breites Themenspektrum aus verschiedenen Bibliotheken und Archiven darstellt, dürfte es erstrebenswert sein, für eine höhere Präsenz über die Landesgrenzen hinaus auch an einem grossen internationalen Portal wie Europeana teilzunehmen. Mit dem sorgfältig strukturierten Minimalset von Metadaten sollte eine Integration gut machbar sein. Es käme sicher dem Bedürfnis nach einer allgemeinen Übersicht

3 Markus Brantl: Das International Image Interoperability Framework (IIIF). Neuer Standard für Zusammenarbeit, Nutzerfreundlichkeit und Forschung. In: Bibliotheksmagazin 2016/1, S. 24-27. 
über das Angebot von digitalisierten Dokumenten entgegen. Bis es soweit ist, erlaubt immerhin die Google-Suche das Auffinden von Begriffen aus den Titeldaten sowie aus den Inhaltsübersichten und in absehbarer Zeit sogar aus den Volltexten. Diese Zugänglichkeit entspricht dem Bestreben nach einer guten Sichtbarkeit bei langfristig ausgerichteter Verfügbarkeit. Die Plattform soll für möglichst viele unterschiedlich ausgerichtete Forschungsprojekte eine zuverlässige Bezugsquelle von qualitativ hochstehenden Digitalisaten sein, die auch eindeutig nachweisbar bleiben. 